\title{
Prognostic vs predictive molecular biomarkers in colorectal cancer: is KRAS and BRAF wild type status required for anti-EGFR therapy?
}

\author{
Sergio Rizzo ${ }^{a \ddagger}$, Giuseppe Bronte ${ }^{a \ddagger}$, Daniele Fanale ${ }^{\mathrm{a}}$, Lidia Corsini ${ }^{\mathrm{a}}$, Nicola Silvestris ${ }^{\mathrm{b}}$, Daniele Santinic, \\ Gaspare Gulotta $^{d}$, Viviana Bazan ${ }^{a}$, Nicola Gebbia ${ }^{a}$, Fabio Fulfaro ${ }^{a}$, Antonio Russo ${ }^{a}$ * \\ a Department of Surgical and Oncological Sciences, Section of Medical Oncology, University of Palermo, Palermo, Italy \\ ${ }^{b}$ Medical and Experimental Oncology Unit, Cancer Institute "Giovanni Paolo II", Bari, Italy \\ ${ }^{c}$ Medical Oncology, Campus Bio-Medico University, Rome, Italy \\ ${ }^{d}$ Department of General Surgery, Urgency, and Organ Transplantation, University of Palermo, Italy
}

\section{A R T I C L E I N F O}

Keywords:

EGFR

KRAS

Driver mutations

Monoclonal antibodies

\begin{abstract}
S U M M A R Y
An important molecular target for metastatic CRC treatment is the epidermal growth factor receptor (EGFR). Many potential biomarkers predictive of response to anti-EGFR monoclonal antibodies (cetuximab and panitumumab) have been retrospectively evaluated, including EGFR activation markers and EGFR ligands activation markers. With regard to the "negative predictive factors" responsible for primary or intrinsic resistance to anti-EGFR antibodies a lot of data are now available. Among these, KRAS mutations have emerged as a major predictor of resistance to panitumumab or cetuximab in the clinical setting and several studies of patients receiving first and subsequent lines of treatment have shown that those with tumors carrying KRAS mutations do not respond to EGFR-targeted monoclonal antibodies or show any survival benefit from such treatments. The role of B-RAF mutations, mutually exclusive with KRAS mutations, in predicting resistance to anti-EGFR mAbs is not yet consolidated. It therefore appears that BRAF mutations may play a strong negative prognostic role and only a slight role in resistance to anti-EGFR Abs.
\end{abstract}

(C) 2010 Elsevier Ltd. All rights reserved.

\section{Introduction}

Colorectal cancer (CRC) is the third most common cause of cancerrelated death, with an incidence of almost a million cases annually in both males and females. ${ }^{1}$ Despite the fact that recent progress in diagnosis and treatment has increased the number of patients who have been completely cured at an early stage of the disease, the prognosis for advanced forms of this cancer is still very poor, with treatment limited to palliation for the vast majority of patients. ${ }^{2}$

The development of colorectal cancer (CRC) is a multistep process brought about by the accumulation of several genetic alterations, including chromosomal abnormality, gene mutations and epigenetic modifications involving several genes regulating proliferation, differentiation, apoptosis and angiogenesis. ${ }^{3,4}$

of the various genetic alterations, only a few are involved in cell growth and will lead to cancer development. This phenomenon therefore, known as 'oncogene addiction' might represent a rationale for molecular target therapy, ${ }^{5}$ possibly helping

\footnotetext{
${ }^{*}$ Corresponding author. Antonio Russo, MD, PhD. Department of Surgical and Oncological Sciences, Section of Medical Oncology, Università di Palermo, Palermo, Italy; Via del Vespro 127, 90127 Palermo, Italy. Tel.: + 39091 6552500; fax: +390916554529. E-mail address: lab-oncobiologia@usa.net (A. Russo).
}

to develop new and targeted treatment options in patients with metastatic CRC. ${ }^{6}$

An important molecular target for metastatic CRC treatment is the epidermal growth factor receptor (EGFR). EGFR is a member of the HER (ErbB) family of receptor tyrosine kinases involved in a variety of signal transduction pathways which are able to promote tumor cell proliferation, angiogenesis, invasion and metastasis in different epithelial malignancies. ${ }^{7}$

In more specific terms, the binding of the epidermal growth factor (EGF) to the extracellular binding site of EGFR activates three major signal transduction pathways, including the RAS-RAF mitogenactivated protein kinase (MAPK), phosphatidylinositol 3-kinase (PI3K) and phospholipase $C$ pathways, causing downstream change in the gene expression profile leading to cancer development. ${ }^{8}$

EGFR is expressed on normal human cells but higher levels of expression of the receptor have also been correlated with malignancy in a variety of cancers, including CRC. ${ }^{9}$

Two predominant classes of EGFR inhibitors have been developed including monoclonal antibodies (mAbs), which target the extracellular domain of EGFR, such as cetuximab, and small molecule tyrosine kinase inhibitors (TKIs), which target the receptor catalytic domain of EGFR, such as gefitinib and erlotinib. ${ }^{10}$ Although both classes of agents show clear antitumor activity, only anti-EGFR

\$These authors contributed equally to this work. 
monoclonal antibodies such as Cetuximab and Panitumumab have been approved for clinical use in metastatic CRC. ${ }^{11}$

These costly and potentially toxic treatments are, however, efficient in only a small percentage of patients, and it is therefore extremely important to identify specific factors which will lead to a clearer definition of those patients who will benefit from anti-EGFR treatments.

\section{Potential positive predictive biomarkers}

The major potential predictive factors of response to cetuximab and/or panitumumab evaluated up till now in literature are molecular factors involved more or less directly in the EGFR signaling pathway. Among these, EGFR protein expression, EGFR gene copy number, EGFR gene mutations, and overexpression of EGFR ligands (such as epiregulin and amphiregulin) have been evaluated in order to select patients who may benefit from EGFRtargeted treatment. ${ }^{12}$

Several clinical trials have been performed in patients with metastatic CRC to relate the level of EGFR protein expression, as determined by immunohistochemistry with sensitivity to anti-EGFR antibodies. The results have demonstrated a lack of association between EGFR detection by immunohistochemistry, and response to EGFR-targeted treatment. Objective responses have been observed in patients with low or high EGFR level expression and since this biomarker has proved to be poorly associated with sensitivity to anti-EGFR antibody, it cannot therefore be considered as an inclusion criterion for patients undergoing treatment with cetuximab. ${ }^{13}$

Activating mutations in the EGFR catalytic domain play an important role in determining responsiveness to anti-EGFR treatment in lung cancer; these alterations are however, rare or absent in CRC and are not significantly associated with clinical response of metastatic CRC to the anti-EGFR monoclonal antibodies. ${ }^{14}$

In a small fraction of CRCs, the overexpression of EGFR is frequently associated with amplification of the gene. The evaluation of the EGFR gene copy number evaluated by quantitative PCR does not seem to correlate with the clinical outcome of patients, whereas the analysis by fluorescence in situ hybridation (FISH) appears to be associated with an increase of treatment response. ${ }^{15}$ The predictive value is, however, uncertain and further studies are therefore required to assess the increase of EGFR gene copy number as a predictive marker of response to anti-EGFR treatment.

The overexpression of alternative EGFR ligands, such epiregulin and amphireguline may promote tumor growth and survival by an autocrine loop. ${ }^{16}$ Several studies have correlated the expression of these ligands with sensitivity to cetuximab monotherapy. The results showed a statistically longer progression free survival (PFS) among patients with high expression of epiregulin. The exclusive use of amphiregulin or epiregulin gene expression profile does not, however, result in the selection of patient populations benefiting from cetuximab treatment. ${ }^{17}$

In order to increase the power of patient selection for anti-EGFR therapy, several studies involving the identification of alternative predictive molecular biomarkers have been conducted.

\section{Biomarkers downstream to EGFR}

EGFR-mediated signaling involves two main intracellular cascades: KRAS, which, activating BRAF, triggers in its turn the mitogenactivated protein kinases (MAPKs) and the membrane localization of the lipid kinase PIK3CA, which counteracts with PTEN and promotes AKT1 phosphorylation, thereby activating a parallel intracellular axis. ${ }^{18}$
KRAS is a proto-oncogene encoding a small $21 \mathrm{kD}$ guanosine triphosphate (GTP)/guanosine diphosphate (GDP) binding protein involved in the regulation of cellular response to many extracellular stimuli. ${ }^{19}$ After binding and activation by GTP, RAS recruits the oncogene RAF, which phosphorylates MAP2K (mitogen-activated protein kinase kinase), initiating the MAPK signaling leading to the expression of the protein involved in cell proliferation, differentiation and survival. ${ }^{20}$ PIK3CA is an oncogene encoding for the $\mathrm{p} 110$ subunit of PI3K, which can be activated via interaction by the RAS protein. ${ }^{21}$

The constitutive activation of signaling pathways downstream of the EGFR by mutations in KRAS, BRAF and PI3KCA and the interaction between these pathways, drive the growth and progression of CRC and provide an escape mechanism which allows the tumors to overcome the pharmacological blockade induced by anti-EGFR molecules. $^{22}$

KRAS is the mostly commonly mutated gene in this pathway. It is mutated in 35-45\% of colorectal adenocarcinomas and this alteration is an early event in colon tumorigenesis. ${ }^{23}$ Up to $90 \%$ of activating KRAS gene mutations are detected in codons $12(70 \%)$ and 13 (30\%), and less in codon 61. Frequently these mutations result in an exchange of different amino acids at the catalytic sites which induce the glicine-to-valine substitution associated with a more aggressive tumor growth. ${ }^{24}$

\section{Negative predictive role of KRAS gene mutations}

KRAS mutations have emerged as a major predictor of resistance to panitumumab or cetuximab in the clinical setting and several studies of patients receiving first and subsequent lines of treatment have shown that those with tumors carrying KRAS mutations do not respond to EGFR-targeted monoclonal antibodies or show any survival benefit from such treatments.

Several studies have been conducted in order to explore the role of KRAS mutations as a predictive biomarker of tumors from patients with metastatic CRC treated with anti-EGFR monoclonal antibody (with or without chemotherapy). ${ }^{25}$

The first study evaluating the correlation between $K$-RAS mutational status in primary tumors and absence of response to treatment with cetuximab or panitumumab, was that of Lievre et al., which involved a cohort study of 30 patients, and reported a link between KRAS mutations and lack of response of metastatic patients to EGFR-targeted monoclonal antibodies.

In this study K-RAS mutations were observed in 13 of the 30 patients enrolled in the study and these mutations were closely associated with response to treatment; none of the mutated tumors responded to cetuximab. Among responders none $(0 / 11)$ presented KRAS mutations, while a mutational status of KRAS was found in $68.4 \%(13 / 19)$ of patients who were non-responders $(\mathrm{p}=0.0003)$. The overall survival of K-RAS wild type patients (WT) was significantly higher compared with those with mutated KRAS (median OS: 16.3 vs 6.9 months, $\mathrm{p}=0.016$ ). ${ }^{26}$

Amado et al., confirmed the negative predictive value of KRAS mutations in a randomized phase III study, comparing the effect of panitumumab monotherapy with best supportive care (BSC) in patients with chemotherapy-refractory metastatic CRC.

The treatment effect on PFS in the WT KRAS group was significantly greater $(P$ 0.0001) than in the mutant group. Median PFS in the WT KRAS group was 12.3 weeks for panitumumab and 7.3 weeks for BSC. The Authors therefore concluded their study confining the panitumumab monotherapy efficacy in metastatic CRC to patients with WT KRAS tumors. ${ }^{27}$ In a randomized study of 572 patients, Karapetis et al. examined the role of KRAS mutations in treatment response, comparing the effect of cetuximab monotherapy with best supportive care (BSC) in patients with chemo-refractory metastatic CRC. 


\begin{tabular}{|c|c|c|c|c|c|c|}
\hline \multirow{2}{*}{ VARIABLES } & \multicolumn{3}{|c|}{ Disease-Free Survival } & \multicolumn{3}{|c|}{ Overall Survival } \\
\hline & HR & $95 \% \mathrm{CL}$ & $\mathrm{P}$ & HR & $95 \% \mathrm{CL}$ & $\mathrm{P}$ \\
\hline \multicolumn{7}{|l|}{ Ki-RAS mut. codon 12} \\
\hline Valine & 1.30 & $1.09-1.54$ & $<0.01$ & 1.29 & $1.08-1.55$ & $<0.01$ \\
\hline Aspartate & 1.04 & $0.89-1.22$ & NS & 0.94 & $0.79-1.11$ & NS \\
\hline Cysteine & 1.11 & $0.85-1.46$ & NS & 1.26 & $0.93-1.62$ & NS \\
\hline Serine & 1.42 & $1.04-1.93$ & NS & 1.20 & $0.86-1.70$ & NS \\
\hline Alanine & 1.21 & $0.89-1.66$ & NS & 1.35 & $0.98-1.87$ & NS \\
\hline \multicolumn{7}{|l|}{ K-RAS mut. codon 13} \\
\hline Aspartate & 0.94 & $0.79-1.12$ & NS & 0.93 & $0.78-1.12$ & NS \\
\hline
\end{tabular}

Fig. 1. The RASCAL study: results of the multivariate analysis.

Cetuximab treatment in WT KRAS patients compared to BSC resulted in a significant increase of the OS (median OS: 9.5 vs 4.8 months; $\mathrm{HR}=0.55,95 \% \mathrm{CI}: 0.41-0.74, \mathrm{P}<0.001$ ) and PFS (median PFS: 3.7 vs 1.9 months; $\mathrm{HR}=0.40,95 \% \mathrm{CI}: 0: 30$ to $0: 54, \mathrm{p}<0.001$ ) while among KRAS mutated patients the differences were not significant in either PFS or OS ( $\mathrm{p}=0.96 \mathrm{p}=0.89$, respectively). ${ }^{28}$

Reports regarding KRAS data from large randomized trials have recently been published, including the first-line phase II study OPUS (Oxaliplatin and Cetuximab in First-Line Treatment of metastatic CRC) and the first-line phase III study CRYSTAL (Cetuximab Combined With Irinotecan in First-Line Therapy for Metastatic Colorectal Cancer). ${ }^{29}$ These results show that the KRAS mutated patients do not benefit from addition of cetuximab to conventional chemotherapy. Both PFS and OS were similar for cetuximab and control groups in patients carrying tumors with KRAS mutations ( progression-free interval $=1.8$ vs 1.8 months $[\mathrm{HR}=0.99,95 \% \mathrm{CI}=$ 0.73 to $1.35, P=0.96$ ]; overall survival $=4.6$ vs 4.5 months $[\mathrm{HR}=0.98$, $95 \% \mathrm{CI}=0.70$ to $1.37, P=0.89])$. In the wild-type KRAS patients, however, cetuximab treatment was associated with statistically significantly $(P<0.001)$ longer survival than control treatment (progression-free interval $=3.7$ vs 1.9 months $[\mathrm{HR}=0.40,95 \% \mathrm{CI}=$ 0.30 to 0.54 ]; overall survival $=9.5$ vs 4.8 months $[\mathrm{HR}=0.55$, $95 \% \mathrm{CI}=0.41$ to $0.74, P<0.001] .^{29,30}$

Retrospective data from the OPUS and CRYSTAL studies indicate that from the addition of cetuximab to first-line FOLFOX (folinic acid, fluorouracil, and oxaliplatin) ${ }^{30}$ or FOLFIRI (folinic acid, fluorouracil and irinotecan $)^{29}$ chemotherapy does not benefit patients with KRAS mutations. In fact, the OPUS study indicates that addition of EGFR-targeted treatment to chemotherapy may even be detrimental in such patients. ${ }^{30}$ In some cases the addition of cetuximab or panitumumab to standard chemotherapy may be not useful even in KRAS wt patients.

In the PACCE (Panitumumab Advanced Colorectal Cancer Evaluation) study, the addition of panitumumab to bevacizumab and chemotherapy was associated with shortening of the progression free interval among patients with tumors carrying WT KRAS (11.5 months in the chemotherapy-bevacizumab arm vs 9.8 months in the panitumumab-chemotherapy-bevacizumab arm). ${ }^{31}$

In the CAIRO-2 (CApecitabine, IRinotecan, and Oxaliplatin trial) study, the addition of cetuximab to capecitabine, oxaliplatin, and bevacizumab as first-line treatment in patients with metastatic CRC had no effect on progression-free interval among those with tumors carrying WT KRAS (10.6 months in the chemotherapy-bevacizumab arm vs 10.5 months in the combined cetuximab arm). ${ }^{32}$

In the large COIN trial the addition of cetuximab to Oxaliplatinbased CT did not improve OS or PFS with increased nonhematological toxicity in KRAS wt patients, even if the very advanced disease in the COIN population may be the reason for the negative results.. ${ }^{33}$

\section{Prognostic role of KRAS gene mutations}

The collaborative RASCAL II studies have been conducted with the aim of investigating the prognostic role of KRAS mutations in CRC progression.

To explore the effect of KRAS mutations at different stages of CRC, 3493 patients were recruited in this multivariate analysis. The results obtained suggest that of the 12 possible mutations on codons 12 and 13 of KRAS, only the substitution glycine to valine on codon 12 , found in $8.6 \%$ of all patients, had a statistically significant impact on PFS ( $P=0.0004$, HR 1.3) and OS $(P=0.008$, HR 1.29) (Fig. 1). Furthermore, these mutations have a greater impact on outcome in Dukes' $\mathrm{C}$ cancers (failure-free survival, $\mathrm{P}=0.008, \mathrm{HR} 1.5$; OS $\mathrm{P}=0.02 \mathrm{HR} 1.45$ ) than in Dukes'B cancers (failure-free survival, $\mathrm{P}=0.46$, HR 1.12; OS $\mathrm{P}=0.36$ HR 1.15).

The RASCAL studies therefore show that tumors carrying mutated KRAS might have an effect on the survival rate of CRC patients, and that the specific codon 12 glycine/valine mutation not only might play a role in the tumor progression, but this alteration might also predispose to more aggressive biological behavior in patients with advanced CRC. ${ }^{23}$

Not all identified mutations of KRAS, however, necessarily have the same biological, biochemical and functional role. ${ }^{34}$

The prognostic role of KRAS mutations in CRC progression is still controversial. In a recent work Roth el al. in accordance with data from smaller retrospective studies (PETACC-3, EORTC 40993, SAKK 60-00) sustain the lack of prognostic value for KRAS mutation status for PFS and OS in patients with CRC. The prognostic significance of KRAS mutations observed in the multivariate analysis of the RASCAL studies might have been overestimated as a result of the number of subset analyses. Larger studies are therefore required in order to confirm whether a specific KRAS mutation might lead to a clinically relevant prognostic effect in patients with CRC. ${ }^{35}$

\section{Predictive and prognostic role of BRAF gene mutations}

The most frequent BRAF mutation observed is a DNA missense mutation leading to a valine to glutamic acid amino acid substitution (V600E). It is functionally the most important mutation involved in the receptor-independent aberrant activation of the MEK-ERK pathway and CRC carcinogenesis. Mutations of BRAF, kinase located downstream of K-RAS in the EGFR signal transduction pathway, are found in colorectal tumors with a relatively low frequency (approximately 10\%). These alterations have been studied in recent years in order to reach a better understanding of its possible role in predicting response to antiEGFR drugs. Assuming that BRAF mutations may have, in K-RAS wild type tumors, a predictive/prognostic role, Di Nicolantonio et al. have retrospectively analyzed RR, TTP (time to progression), OS and mutational status of $K-R A S$ and BRAF in 113 patients 


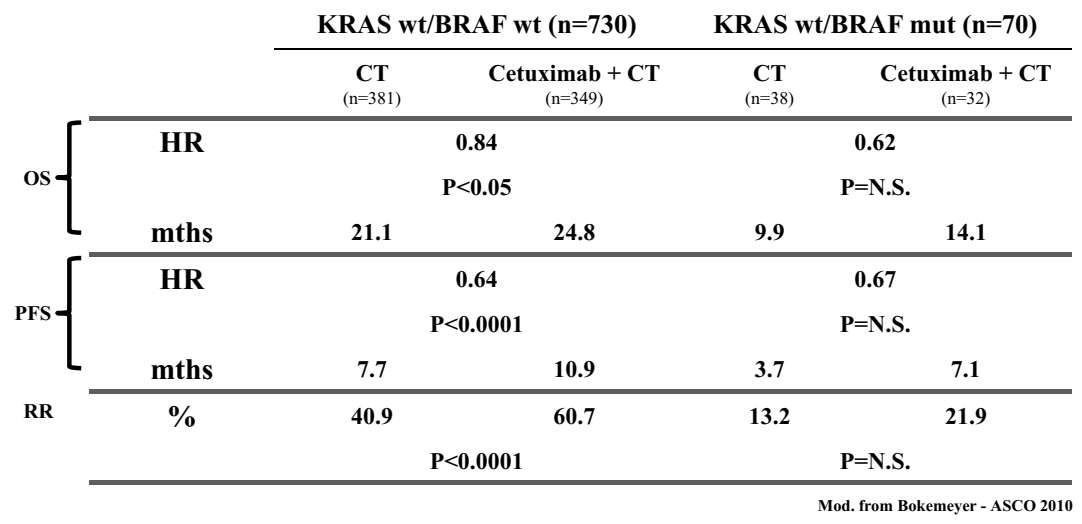

Fig. 2. B-RAF mutational status in K-RAS Wt patients: pooled analysis of CRYSTAL and OPUS studies.

with mCRC treated with cetuximab or panitumumab. The BRAF mutation V600E was the only one present in the cohort of analyzed patients. Its presence appears inversely related to the activity of the treatment, especially since some of the mutated patients (11 out of $79 \mathrm{~K}-R A S$ w.t.) responded to the drug administration and conversely none of the responders presented the mutation in question ( $\mathrm{p}=0.029)$. In addition, $B R A F$-mutated patients compared with w.t obtained a significantly shorter PFS $(\mathrm{p}=0.011)$ and OS $(\mathrm{p}<0.0001)$. The authors concluded by supporting the need to have $B R A F$ w.t. in order to obtain response from treatment with EGFR inhibitors. ${ }^{36}$ Furthermore, in BRAF-mutated colorectal cancer cell lines, the sensitivity to EGFR inhibitors may be restored by means of the multikinase inhibitor sorafenib. Several studies have confirmed the negative prognostic role of BRAF mutations. In $K-R A S$ wild-type patients, $B R A F$-mutated individuals have shown a worse outcome in terms of PFS and OS. Furthermore, BRAF is prognostic for OS, especially in patients with microsatellite instability (MSI) low (MSI-L) and stable (MSI-S) tumors. In the MSI-H (high) subpopulation no prognostic value of $K-R A S$ and BRAF mutation status was found for RFS and OS. ${ }^{35}$ The relatively low frequency of this genetic alteration in colorectal cancer makes it rather difficult to draw absolute conclusions also based on post-hoc analysis of the Phase II and III studies recently published. The retrospective analysis of the B-RAF mutational status has been performed in pts in the CRYSTAL and OPUS studies (Fig. 2). The analysis of the OS and PFS in pts with wt KRAS/wt BRAF showed a significant difference between the two treatment arms. This difference was not significant in the mutated BRAF/KRAS wt patients. Nevertheless, these patients seem to benefit from the addition of Cetuximab, with an increase of OS and a doubling of PFS rates. Furthermore, there is clearly a worse outcome in mutated BRAF patients independently of treatment with Cetuximab, which supports the hypothesis of a possible negative prognostic role of BRAF mutations. ${ }^{37}$ In the CAIRO-2 study, a similar pattern was observed in a large series of $\mathrm{MCRC}$ patients treated with chemotherapy and Bevacizumab with or without Cetuximab. It was seen that the BRAF mutation is associated with a worse outcome, both in terms of PFS and of OS, independently of the addition of Cetuximab to the treatment.. ${ }^{38}$ To date, therefore, the negative value of mutations of $B R A F$ is only suggested by some reports, ${ }^{39}$ while the significant negative prognostic value seems to be now established. ${ }^{40}$

\section{PTEN-PI3K-AKT-mTOR pathway alterations}

In addition to KRAS and BRAF, the HER family of receptors also activates the PI3K signaling pathway, which in turn can be oncogenically deregulated either by activating mutations in the PIK3CA p110 subunit or by inactivation of the PTEN phosphatase. The role of deregulated PIK3CA/PTEN signaling on the response to targeted therapy has therefore been investigated in breast cancer, ${ }^{41}$ glioblastoma ${ }^{42}$ and also mCRC. Mutation constitutive activation of the PI3K signaling pathway has been reported to occur in $~ 30 \%$ of colon tumors, primarily due to activating mutations in exons 9 and 20 of the PIK3CA gene ${ }^{43,44}$ and, to a lesser extent, due to inactivating PTEN mutations or PTEN promoter methylation. ${ }^{45}$ PTEN is a tumor suppressor that acts as a negative regulator of PI3K signaling by converting PIP3 to PIP2, and truncating mutations which result in loss of PTEN expression, reported in $20 \%$ of MSI colon cancers. ${ }^{46-51}$

The molecular alteration of PTEN is often caused by epigenetic mechanisms, ${ }^{45}$ supporting the detection of the intact protein by IHC as a better diagnostic tool than gene sequencing, as it potentially covers more mechanisms of alteration. PIK3CA mutation and PTEN expression status predicts response of colon cancer cells to the EGFR inhibitor cetuximab distinguishing drug sensitive and resistant cell lines. Colon cancer cell lines with activating PIK3CA mutations or loss of PTEN expression (PTEN null) were more resistant to cetuximab than PIK3CA wild type (WT)/PTEN expressing cell lines. Furthermore, cell lines that were PIK3CA mutant/PTEN null and Ras/BRAF mutant were highly resistant to cetuximab compared with those without dual mutations/PTEN loss, indicating that constitutive and simultaneous activation of the Ras and PIK3CA pathways confer maximal resistance to this agent. On the other hand, these patients may be suitable candidates for treatment with newer targeted drugs currently involved in clinical trials, which inhibit signaling mediators further downstream, including PI3K, AKT, or mTOR inhibitors and Ras, Raf, or MEK inhibitors. A possible mechanism of resistance to cetuximab of these cell lines may be the existence of alternate mutations in the Ras/BRAF and or PIK3CA/PTEN pathway. Increased sensitivity to cetuximab was observed in PIK3CA WT lines. Likewise, breast cancers with either activating mutations in PIK3CA or with loss of PTEN expression respond poorly to treatment with the Her2/Neu targeting antibody, trastuzumab. ${ }^{52}$ Consistent with the present findings, Frattini et al. recently reported that colon tumors with loss of PTEN expression have significantly reduced response to cetuximab. ${ }^{48}$ Furthermore, PIK3CA mutations and PTEN loss in colorectal tumors are statistically and significantly associated with lack of response to panitumumab or cetuximab treatment. ${ }^{18}$ PIK3CA mutations and/or loss of PTEN expression are negatively associated with PFS, and loss of PTEN expression is also linked with poorer OS. A priori screening of colon tumors for PTEN expression status and PIK3CA and Ras/BRAF mutation status could help stratify patients likely to benefit from this therapy. ${ }^{53}$ Razis et al. ${ }^{54}$ reported that normal PTEN protein expression was associated with a higher response rate and longer time to progression in patients treated with cetuximabbased therapy, despite a 50\% response rate observed in patients who had lost PTEN protein expression. Loupakis et al. performed a retrospective analysis on the status of PTEN in a cohort of 


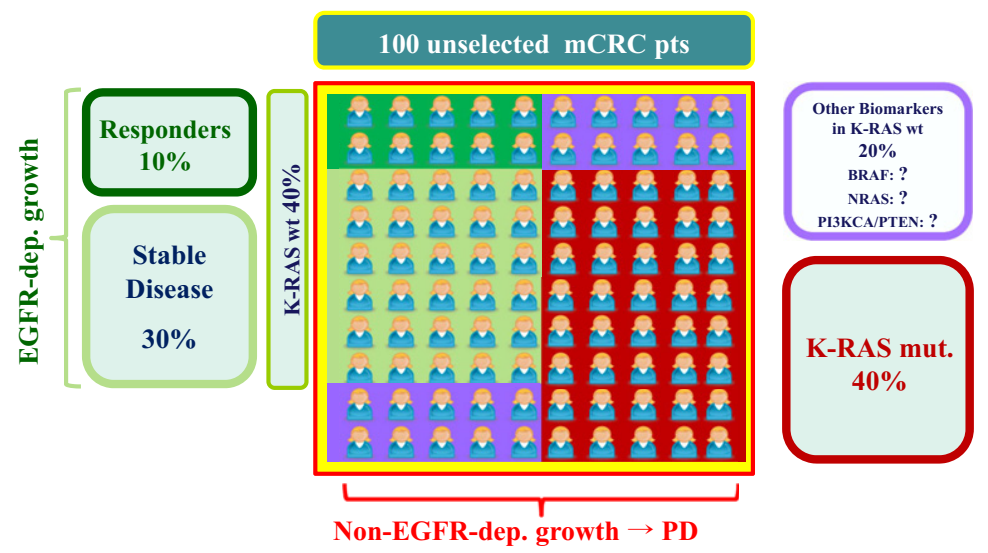

Fig. 3. Activity of anti EGFR-Ab monotherapy in chemorefractory mCRC patients.

55 metastases from patients with irinotecan refractatory mCRC treated with irinotecan and cetuximab: 12 (36\%) of 33 patients with PTEN-positive metastases were responders compared with one (5\%) of 22 who had PTEN-negative metastases. ${ }^{50}$ Patients with PTENpositive metastases and KRAS wild type had longer PFS compared with other patients.

Collectively, these findings show that colon cancer cell lines with constitutively active PI3K signaling are refractory to cetuximab. These data imply that colon cancer cell lines which acquire mutations that result in constitutive activation of the PI3K pathway have a diminished dependence on canonical EGFR ligand-induced signaling for their growth and are, therefore, more resistant to EGFR targeted therapies. Collectively, these studies provide additional clinical evidence that the mutation status of the PI3K signaling pathway should be considered before treatment with EGFR family antagonists. Colon cancer cell lines mutant for PIK3CA/PTEN null are significantly more resistant to cetuximab compared with PIK3CA/PTEN WT lines. Furthermore, cell lines with both constitutively active PIK3CA and Ras/BRAF signaling were highly refractory to cetuximab. These studies suggest that combining mutation analysis for K-RAS and PIK3CA (loss of PTEN and/or PIK3CA mutation) could identify up to $70 \%$ of patients with metastatic colorectal cancer who are unlikely to respond to treatment with an EGFR-targeted monoclonal antibody. ${ }^{18}$

\section{Conclusions}

EGFR and its downstream K-RAS/B-RAF and PTEN-PI3K-AKT-mTOR pathways play an important role in tumorigenesis and tumor progression of CRC.

Only in a fraction of patients with mCRC (10\% according to RECIST criteria that are not adequate for the evaluation of tumor response to new molecular agents) anti-EGFR mAbs (cetuximab and panitumumab) have shown remarkable efficacy (Fig. 3). Forty percent of patients with mCRC obtain clinical benefit from monotherapy with anti-EGFR antibodies, which means that they present EGFR-dependent tumoral growth. The remaining 60\% of the patients are Non Responders, that is, they present tumoral growth which does not depend on the EGFR block mediated by the anti-EGFR Abs. This limitation seems linked mainly to oncogenic KRAS mutations in codon 12 and 13, which implies its continuous activation and signal transduction to the nucleus, even when the EGFR is blocked. KRAS mutation seems to be responsible in $35-45 \%$ cases of resistance to anti-EGFR Abs. The potential bias associated with a retrospective evaluation of the mutational status of KRAS in the CRYSTAL and OPUS studies seems not relevant today, since in both studies the difference between the ITT population and the population assessable for KRAS is negligible.
Another group of patients, representing $10 \%$ of the total number of patients, carry the BRAF mutation that is mutually exclusive with $K R A S$ mutations. It therefore appears that BRAF mutations may play a strong negative prognostic role and only a slight role in resistance to anti-EGFR Abs, since even pts with mCRCs treated without Cetuximab have significantly reduced PFS and OS when the tumor presents a BRAF mutation. The investigation of other biomarkers such as EGFR copy number and expression levels of EGFR ligands, phosphatase and tensin homolog (PTEN) loss or NRAS mutation may be useful to further refine the responder population. Nevertheless, up till now, clinical evidence all points towards the identification of the KRAS mutation as the only evaluated and reproducible predictive factor of resistance to anti-EGFR antibodies.

\section{Conflict of interests}

All authors have no conflict of interest to declare.

\section{References}

1. Parkin DM, Bray F, Ferlay J, Pisani P. Global cancer statistics, 2002. CA Cancer J Clin 2005;55:74-108.

2. Carpenter SG, Carson J, Fong Y. Regional liver therapy using oncolytic virus to target hepatic colorectal metastases. Semin Oncol 2010;37:160-9.

3. Russo A, Rizzo S, Bronte G, et al. The long and winding road to useful predictive factors for anti-EGFR therapy in metastatic colorectal carcinoma: the KRAS/BRAF pathway. Oncology 2009;77(Suppl 1):57-68.

4. Vogelstein B, Fearon ER, Hamilton SR, et al. Genetic alterations during colorectaltumor development. N Engl J Med 1988;319:525-32.

5. Weinstein IB, Joe A. Oncogene addiction. Cancer Res 2008;68:3077-80; discussion 80.

6. Amador ML, Hidalgo M. Epidermal growth factor receptor as a therapeutic target for the treatment of colorectal cancer. Clin Colorectal Cancer 2004;4:51-62.

7. Tedesco KL, Lockhart AC, Berlin JD. The epidermal growth factor receptor as a target for gastrointestinal cancer therapy. Curr Treat Options Oncol 2004;5:393403.

8. Harari PM. Epidermal growth factor receptor inhibition strategies in oncology. Endocr Relat Cancer 2004;11:689-708.

9. Harding J, Burtness B. Cetuximab: an epidermal growth factor receptor chimeric human-murine monoclonal antibody. Drugs Today (Barc) 2005;41:107-27.

10. Lurje G, Lenz HJ. EGFR signaling and drug discovery. Oncology 2009;77:400-10.

11. Vokes EE, Chu E. Anti-EGFR therapies: clinical experience in colorectal, lung, and head and neck cancers. Oncology (Williston Park) 2006;20:15-25.

12. Spindler KL, Lindebjerg J, Nielsen JN, et al. Epidermal growth factor receptor analyses in colorectal cancer: a comparison of methods. Int J Oncol 2006;29: 1159-65.

13. Chung KY, Shia J, Kemeny NE, et al. Cetuximab shows activity in colorectal cancer patients with tumors that do not express the epidermal growth factor receptor by immunohistochemistry. J Clin Oncol 2005;23:1803-10.

14. Moroni M, Sartore-Bianchi A, Benvenuti S, Artale S, Bardelli A, Siena S. Somatic mutation of EGFR catalytic domain and treatment with gefitinib in colorectal cancer. Ann Oncol 2005;16:1848-9.

15. Ooi A, Takehana T, Li X, et al. Protein overexpression and gene amplification of HER-2 and EGFR in colorectal cancers: an immunohistochemical and fluorescent in situ hybridization study. Mod Pathol 2004;17:895-904. 
16. Jacobs B, De Roock W, Piessevaux H, et al. Amphiregulin and epiregulin mRNA expression in primary tumors predicts outcome in metastatic colorectal cancer treated with cetuximab. J Clin Oncol 2009;27:5068-74.

17. Khambata-Ford S, Garrett CR, Meropol NJ, et al. Expression of epiregulin and amphiregulin and K-ras mutation status predict disease control in metastatic colorectal cancer patients treated with cetuximab. J Clin Oncol 2007;25:3230-7.

18. Sartore-Bianchi A, Martini M, Molinari F, et al. PIK3CA mutations in colorectal cancer are associated with clinical resistance to EGFR-targeted monoclonal antibodies. Cancer Res 2009;69:1851-7.

19. Schubbert S, Shannon K, Bollag G. Hyperactive Ras in developmental disorders and cancer. Nat Rev Cancer 2007;7:295-308

20. Bos JL. ras oncogenes in human cancer: a review. Cancer Res 1989;49:4682-9.

21. Yarden Y, Sliwkowski MX. Untangling the ErbB signalling network. Nat Rev Mol Cell Biol 2001;2:127-37.

22. Siena S, Sartore-Bianchi A, Di Nicolantonio F, Balfour J, Bardelli A. Biomarkers predicting clinical outcome of epidermal growth factor receptor-targeted therapy in metastatic colorectal cancer. J Natl Cancer Inst 2009;101:1308-24.

23. Andreyev HJ, Norman AR, Cunningham D, et al. Kirsten ras mutations in patients with colorectal cancer: the 'RASCAL II' study. Br J Cancer 2001;85:692-6.

24. Boughdady IS, Kinsella AR, Haboubi NY, Schofield PF. K-ras gene mutation in colorectal adenomas and carcinomas from familial adenomatous polyposis patients. Surg Oncol 1992;1:269-74.

25. Benvenuti S, Sartore-Bianchi A, Di Nicolantonio F, et al. Oncogenic activation of the RAS/RAF signaling pathway impairs the response of metastatic colorectal cancers to anti-epidermal growth factor receptor antibody therapies. Cancer Res 2007;67:2643-8.

26. Lievre A, Bachet JB, Le Corre D, et al. KRAS mutation status is predictive of response to cetuximab therapy in colorectal cancer. Cancer Res 2006;66: 3992-5

27. Amado RG, Wolf $M$, Peeters $M$, et al. Wild-type KRAS is required for panitumumab efficacy in patients with metastatic colorectal cancer. J Clin Oncol 2008;26:1626-34.

28. Karapetis CS, Khambata-Ford S, Jonker DJ, et al. K-ras mutations and benefit from cetuximab in advanced colorectal cancer. N Engl J Med 2008;359:1757-65.

29. Van Cutsem E, Kohne $\mathrm{CH}$, Hitre E, et al. Cetuximab and chemotherapy as initial treatment for metastatic colorectal cancer. N Engl J Med 2009;360:1408-17.

30. Bokemeyer C, Bondarenko I, Makhson A, et al. Fluorouracil, leucovorin, and oxaliplatin with and without cetuximab in the first-line treatment of metastatic colorectal cancer. J Clin Oncol 2009;27:663-71.

31. Hecht JR, Mitchell E, Chidiac T, et al. A randomized phase IIIB trial of chemotherapy, bevacizumab, and panitumumab compared with chemotherapy and bevacizumab alone for metastatic colorectal cancer. J Clin Oncol 2009;27:672-80.

32. Tol J, Koopman M, Cats A, et al. Chemotherapy, bevacizumab, and cetuximab in metastatic colorectal cancer. N Engl J Med 2009;360:563-72.

33. Maughan TS, Adams R, Smith CG, et al. Identification of potentially responsive subsets when cetuximab is added to oxaliplatin-fluoropyrimidine chemotherapy $(\mathrm{CT})$ in first-line advanced colorectal cancer (aCRC): Mature results of the MRC COIN trial. J Clin Oncol 2010;28:15s:abstr 3502.

34. Bazan V, Migliavacca M, Zanna I, et al. Specific codon $13 \mathrm{~K}$-ras mutations are predictive of clinical outcome in colorectal cancer patients, whereas codon 12 K-ras mutations are associated with mucinous histotype. Ann Oncol 2002;13: 1438-46.

35. Roth AD, Tejpar S, Delorenzi M, et al. Prognostic role of KRAS and BRAF in stage II and III resected colon cancer: results of the translational study on the PETACC-3, EORTC 40993, SAKK 60-00 trial. J Clin Oncol 2010;28:466-74.
36. Di Nicolantonio F, Martini M, Molinari F, et al. Wild-type BRAF is required for response to panitumumab or cetuximab in metastatic colorectal cancer. J Clin Oncol 2008;26:5705-12.

37. Bokemeyer C, Kohne C, Rougier P, Stroh C, Schlichting M, Van Cutsem E. Cetuximab with chemotherapy (CT) as first-line treatment for metastatic colorectal cancer (mCRC): Analysis of the CRYSTAL and OPUS studies according to KRAS and BRAF mutation status. J Clin Oncol 2010;28:15s:abstr 3506.

38. Tol J, Nagtegaal ID, Punt CJ. BRAF mutation in metastatic colorectal cancer. N Engl J Med 2009;361:98-9.

39. Loupakis F, Ruzzo A, Cremolini C, et al. KRAS codon 61, 146 and BRAF mutations predict resistance to cetuximab plus irinotecan in KRAS codon 12 and 13 wildtype metastatic colorectal cancer. Br J Cancer 2009;101:715-21.

40. Souglakos J, Philips J, Wang R, et al. Prognostic and predictive value of common mutations for treatment response and survival in patients with metastatic colorectal cancer. Br J Cancer 2009;101:465-72.

41. Nagata Y, Lan KH, Zhou X, et al. PTEN activation contributes to tumor inhibition by trastuzumab, and loss of PTEN predicts trastuzumab resistance in patients. Cancer Cell 2004;6:117-27.

42. Mellinghoff IK, Wang MY, Vivanco I, et al. Molecular determinants of the response of glioblastomas to EGFR kinase inhibitors. N Engl J Med 2005;353:2012-24.

43. Barault L, Veyrie $\mathrm{N}$, Jooste $\mathrm{V}$, et al. Mutations in the RAS-MAPK, PI(3)K (phosphatidylinositol-3-OH kinase) signaling network correlate with poor survival in a population-based series of colon cancers. Int J Cancer 2008;122: 2255-9.

44. Samuels Y, Wang Z, Bardelli A, et al. High frequency of mutations of the PIK3CA gene in human cancers. Science 2004;304:554.

45. Goel A, Arnold CN, Niedzwiecki D, et al. Frequent inactivation of PTEN by promoter hypermethylation in microsatellite instability-high sporadic colorectal cancers. Cancer Res 2004;64:3014-21.

46. Guanti G, Resta N, Simone C, et al. Involvement of PTEN mutations in the genetic pathways of colorectal cancerogenesis. Hum Mol Genet 2000;9:283-7.

47. Shin KH, Park YJ, Park JG. PTEN gene mutations in colorectal cancers displaying microsatellite instability. Cancer Lett 2001;174:189-94.

48. Frattini M, Saletti P, Romagnani E, et al. PTEN loss of expression predicts cetuximab efficacy in metastatic colorectal cancer patients. $\mathrm{Br} \mathrm{J}$ Cancer 2007;97:1139-45.

49. Laurent-Puig P, Cayre A, Manceau G, et al. Analysis of PTEN, BRAF, and EGFR status in determining benefit from cetuximab therapy in wild-type KRAS metastatic colon cancer. J Clin Oncol 2009;27:5924-30.

50. Loupakis F, Pollina L, Stasi I, et al. PTEN expression and KRAS mutations on primary tumors and metastases in the prediction of benefit from cetuximab plus irinotecan for patients with metastatic colorectal cancer. J Clin Oncol 2009; 27:2622-9.

51. Sartore-Bianchi A, Di Nicolantonio F, Nichelatti M, et al. Multi-determinants analysis of molecular alterations for predicting clinical benefit to EGFR-targeted monoclonal antibodies in colorectal cancer. PLoS One 2009;4:e7287.

52. Berns K, Horlings HM, Hennessy BT, et al. A functional genetic approach identifies the PI3K pathway as a major determinant of trastuzumab resistance in breast cancer. Cancer Cell 2007;12:395-402.

53. Jhawer M, Goel S, Wilson AJ, et al. PIK3CA mutation/PTEN expression status predicts response of colon cancer cells to the epidermal growth factor receptor inhibitor cetuximab. Cancer Res 2008;68:1953-61.

54. Razis E, Briasoulis E, Vrettou E, et al. Potential value of PTEN in predicting cetuximab response in colorectal cancer: an exploratory study. BMC Cancer 2008;8:234. 